\title{
Letter to Editor re (2020 Sep;95(4):564-570)
}

\author{
Christian Zammit $^{1}$ (1) $\cdot$ Pierre Schembri Wismayer $^{1} \cdot$ Jean Calleja Agius ${ }^{1}$
}

Received: 28 June 2021 / Accepted: 22 July 2021 / Published online: 4 August 2021

(c) Japanese Association of Anatomists 2021

\section{Dear Editor,}

We have read the article "Thiel's embalming method with additional intra-cerebral ventricular formalin injection (TEIF) for cadaver training of head and brain surgery" published in your journal [2020 Sep;95(4):564-570]. We want to share our personal experience at the Anatomy Department, Faculty of Medicine and Surgery, University of Malta.

We are privileged that in our department, we have a dissection hall, whereby undergraduate students and postgraduate medical professionals can augment their learning experience using cadaveric dissection. About 15 years ago, we changed our embalming system from a formalin-based one, to Thiel Embalming. We experienced a considerable improvement the preservation of organs and tissues, with regards to colour, consistency, natural flexibility, natural plasticity and transparency. It offers a realistic simulation of a life model, which benefitted mostly surgical trainees to refine their surgical skills. In fact, our department regularly holds national and international conferences for a wide range of surgical specialities based on cadaveric dissection.

Despite the many advantages of Thiel Embalming, in accordance with the present study, we found that preservation of the central nervous system was not adequate with this embalming method, and neurosurgical procedures were unsuccessful. We thus opted to start injecting 10\% formalin from each orbit, through the superior orbital fissure, in conjunction with direct injection into the brain parenchyma via 2 burr holes through each parietal lobe. This resulted in partial preservation of the cerebral hemispheres but cause direct injury to the brain parenchyma at the site of injection. To limit such injury, we started injecting the formalin into the subdural space via 2 burr holes in each parietal bone, and another 2 small burr holes in the occipital bone to preserve the brainstem and cerebellum. This improved brain preservation and limited traumatic injury at the site of injection. We firmly believe that coupling our technique with the one presented in this study would enable us to have even better preservation of the cerebral tissue.

Interestingly enough, despite forming part of the central nervous systems, we found that the spinal cord is well preserved with the Thiel embalming method, and we had successful neurosurgical procedures on the vertebral column and spinal cord using Thiel embalmed cadavers without any formalin additives. Shedding light on the difference in preservation between the cerebral hemispheres and the spinal cord might help us to elucidate new improved methods to preserve the various components of the central nervous system in the nearby future.

\section{Declarations}

Conflict of interest No potential conflict of interest is reported by the authors.

Publisher's Note Springer Nature remains neutral with regard to jurisdictional claims in published maps and institutional affiliations.
Christian Zammit

christian.m.zammit@um.edu.mt

1 Faculty of Medicine and Surgery, Anatomy Department, University of Malta, Msida, Malta 